\title{
ANÁLISIS DEL ENTORNO EDUCATIVO Y DE LAS MEDIACIONES TECNOLÓGICAS EN LA ENSEÑANZA VIRTUAL DE LAS CIENCIAS MORFOLÓGICAS EN PANDEMIA
}

Analysis of the educational environment and technological mediations in the virtual teaching of Morphological Sciences in pandemic

Fecha de Recepción: 6 de noviembre 2020
Análise do ambiente educacional e das mediações tecnológicas no ensino virtual de Ciências Morfológicas em pandemia

Aceptado para su publicación: 22 de febrero 2021
Javier Elías Fernández Calderón1, 2,a, Mario Aníbal Sambrizzi Vicenzotti ${ }^{2, b}$

1. Departamento de Biología Bucal. Departamento de Patología Bucal. Facultad de Odontología. Universidad Nacional de Córdoba. República Argentina.

2. Área de Imagenología y Diagnóstico Digital. Facultad de Odontología. Universidad Nacional de Córdoba. Argentina. a. Doctor en Odontología (Universidad Nacional de Córdoba)

b. Licenciado en Producción de Bioimágenes (Universidad Nacional de Córdoba) Correspondencia: Fernández, Javier Elías Haya de la Torre S/N Ciudad Universitaria. Córdoba. Argentina. $+540351433-3080$

Correo electrónico: javdens555@hotmail.com

Conflicto de intereses: los autores declaran no tener conflictos de interés.

Fuente de financiamiento: autofinanciado.

\section{Resumen}

Los medios tecnológicos necesarios para el aprendizaje virtual se transformaron en protagonistas de la enseñanza en tiempos de pandemia Covid-19. El objetivo del presente trabajo fue analizar la información referente a las condiciones en las cuales los alumnos desarrollaron las actividades virtuales en el marco actual de aislamiento. Se realizó una encuesta anónima y voluntaria entre los alumnos de un curso de Anatomía Radiológica Oro-Maxilo-Facial de la Facultad de Odontología, Universidad Nacional de Córdoba. El 56\% de los estudiantes estaba en la ciudad de Córdoba y el 64\% vivía con otras personas. La mayoría de los estudiantes contó con los dispositivos necesarios y conexión a internet. El 52\% compartió estos dispositivos y el $40 \%$ manifestó no tener buena conectividad. El $64 \%$ consideró deficiente esta nueva modalidad de enseñanza virtual. Este trabajo puso en evidencia las dificultades de la educación en línea ocasionadas por la desigual disponibilidad de medios tecnológicos y accesibilidad a internet, como así también por las distintas competencias para el uso de las tecnologías de la comunicación y de la información.

Palabras clave: educación a distancia; anatomía; radiología; odontología; infecciones por coronavirus (fuente: DeCS BIREME).

\footnotetext{
Abstract

The necessary technological means for virtual learning have become the protagonists of teaching in times of the Covid-19 pandemic. The aim of the present work was to analyze the information regarding the conditions in which students developed virtual activities in the current isolation context. An anonymous and voluntary survey was carried out among the students of a course of Oro-Maxillo-Facial Radiological Anatomy of the
} 
School of Dentistry, Universidad Nacional de Córdoba. $56 \%$ percent of the students were in the city of Córdoba and $64 \%$ lived with other people. Most of the students had the necessary devices and internet connection. 52\% percent shared these devices and $40 \%$ said they did not have good connectivity. $64 \%$ percent considered this new virtual teaching modality to be deficient. This work highlighted the difficulties of online education caused by the unequal availability of technological means and Internet accessibility, as well as by the different competencies in the use of communication and information technologies.

Key words: education, distance; anatomy; radiology; dentistry; coronavirus infections (source: MeSH NLM).

\section{Resumo}

O ensino universitário na pandemia de covid-19 teve que se adaptar à virtualidade. Os meios tecnológicos necessários para a aprendizagem virtual (hardware, software) tornaram-se protagonistas do ensino em tempos de pandemia. O objetivo deste trabalho foi coletar $e$ analisar informações sobre as condições em que os alunos desenvolveram atividades virtuais no atual quadro de isolamento. Foi realizada uma pesquisa anônima e voluntária entre os alunos de um curso de Anatomia Radiológica Oro-Maxilo-Facial da Facultad de Odontologia. Universidad Nacional de Córdoba. 56\% dos alunos residia na cidade de Córdoba. 64\% viviam com outras pessoas, o que prejudicou a concentração ao assistir às aulas virtuais. A maioria dos alunos possuía os aparelhos e conexão à Internet necessários. 52\% compartilharam esses dispositivos e a conexão com a internet não teve uma boa avaliação em $40 \%$ dos casos. $64 \%$ consideram esta nova modalidade de ensino virtual deficiente. A migração da sala de aula para a casa do aluno afetou sua concentração nas aulas virtuais. Todos os alunos tinham um dispositivo, embora a conexão com a Internet não fosse boa. Embora os alunos tenham ficado satisfeitos com o processo, mais da metade o classificou como deficiente. Esses dados permitem inferir que existem determinadas situações que devem ser conhecidas e consideradas no âmbito do ensino online para eliminar a exclusão digital e criar inclusão digital em um ambiente virtual eficaz.

Palavras-chave: educação a distancia; anatomía; radiologia; odontologia; infecções por coronavirus (fonte: DeCS BIREME).

\section{Introducción}

El presente siglo XXI está marcado por el auge de la era digital y las modernas tecnologías que impulsan el advenimiento de la llamada $4^{\text {ta }}$ revolución industrial con la aparición de la biotecnología, la robótica, la inteligencia artificial, la realidad aumentada y la web 4.0. En este contexto nuestros alumnos universitarios son jóvenes prosumidores, nativos digitales 2.0 acostumbrados a la producción, intercambio y consumo de contenido en entornos digitales interactivos que presentan distintos tipos de alfabetización digital y transmedia en muchos casos acorde a su nivel socio-económico y cultural ${ }^{1,2}$.

Por otra parte, las innovaciones tecnológicas y la sociedad de la información constituyen grandes avances científico-tecnológicos que plantean grandes dificultades en el actual modelo educativo y promueven profundos cambios en la educación formal para que se adopten y apliquen sistemas y técnicas de enseñanza - aprendizaje diferentes a los actuales $^{3,4}$. En el campo de las Ciencias de la Salud el desarrollo de modelos educativos virtuales o en línea que se fundamentan en modernos paradigmas pedagógicos fomentan en el estudiante un pensamiento crítico, constructivo y colaborativo que le hacen apto para la toma de decisiones y la resolución de problemas como futuro profesional de la salud ${ }^{5-7}$.

El brote de Covid-19, enfermedad infecciosa causada por un nuevo coronavirus que estalló en China en 2019, es actualmente una pandemia global que afecta a los países de todo el mundo. Debido a esta pandemia las clases presenciales en la Universidad Nacional de Córdoba (UNC) fueron suspendidas y se debió trasladar la enseñanza a la virtualidad. En este contexto instituciones, docentes y alumnos tuvieron que adaptarse a una nueva situación de enseñanza - aprendizaje. La utilización de recursos didácticos virtuales y la creación de un entorno virtual de aprendizaje efectivo y accesible desde cualquier sitio y dispositivo fueron los retos de esta adaptación de la presencialidad a la virtualidad. Sin embargo, hay un aspecto que es trascendental en este proceso de educación universitaria en línea que se relaciona con los medios tecnológicos que los estudiantes deben disponer para el aprendizaje virtual tales como el hardware (notebook, celular, tablet, memoria Ram), el software, la conexión a internet 
REFO

VOL. XIV | N 1| AÑO 2021

ISSN 1668-7280

ISSN-E 2683-7986 (disponibilidad, ancho de banda), el conocimiento y manejo de las distintas herramientas digitales (plataformas de enseñanza, de videoconferencia, etc) y hasta los más básicos como son el ambiente físico y el suministro eléctrico adecuados ${ }^{8}$.

Los objetivos del presente trabajo fueron: a) Obtener y analizar información referente a las condiciones del entorno en el cual los alumnos desarrollaron las actividades virtuales de la enseñanza de las Ciencias Morfológicas debido a la pandemia Covid-19 durante el ciclo lectivo 2020 en la UNC, b) Determinar el impacto de las condiciones socio-económico-culturales y ambientales en el proceso de enseñanza-aprendizaje en el marco actual de aislamiento y c) Conocer y contemplar determinados aspectos de la educación virtual en el actual contexto para mejorar el proceso educativo.

\section{Materiales y Métodos}

Se realizó una encuesta anónima y voluntaria entre alumnos $(n=50)$ de un curso de Diagnóstico por imágenes para la enseñanza de la Anatomía Radiológica oro-maxilo-facial en la Facultad de Odontología de la UNC. Para la encuesta se utilizó la herramienta de formularios Google Forms (Formularios de Google) que es un software de administración de encuestas gratuito con la cuenta de Google y que se encuentra disponible a través del servicio de alojamiento de archivos Google Drive o en Google Docs. A través del editor de formularios se empleó la metodología de preguntas de opciones múltiples (se selecciona una sola opción), respuesta corta y casillas (se pueden seleccionar varias opciones). Se realizó un estudio transversal y descriptivo del análisis estadístico y la interpretación de los datos obtenidos.

\section{Resultados}

El total de los alumnos encuestados fue de nacionalidad argentina, siendo el $80 \%$ mujeres y el $20 \%$ hombres. El $36 \%$ vivía en la provincia de Córdoba (el $24 \%$ era de la ciudad de Córdoba) y el $64 \%$ en otras provincias. El $56 \%$ se encontró viviendo en la ciudad de Córdoba sede de la Facultad de Odontología de la UNC.

El $36 \%$ vivía solo, el $56 \%$ con la familia y el $8 \%$ con otros estudiantes. En cuanto a si vivir con otras personas afectaba su concentración al momento de presenciar las clases virtuales el $60 \%$ consideró que sí, mientras que el $32 \%$ expresó que no y un $8 \%$ no expresó opinión al respecto. El $68 \%$ de los estudiantes dispuso de un espacio físico adecuado para presenciar las clases virtuales mientras que el $32 \%$ restante no disponía del mismo (Figura 1).
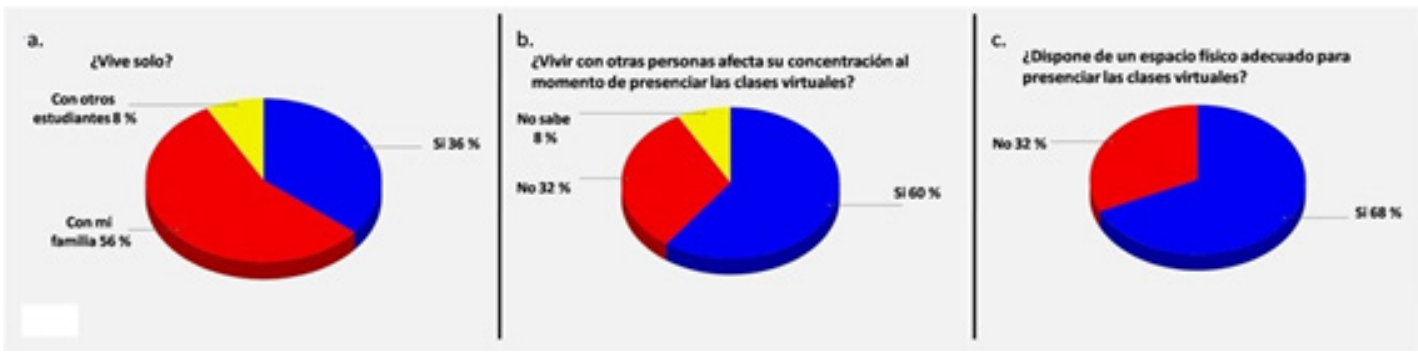

Figura 1. Ilustra: a) con quienes viven los alumnos, b) como esto afectó su concentración y c) disposición de un espacio adecuado para presenciar las clases virtuales.
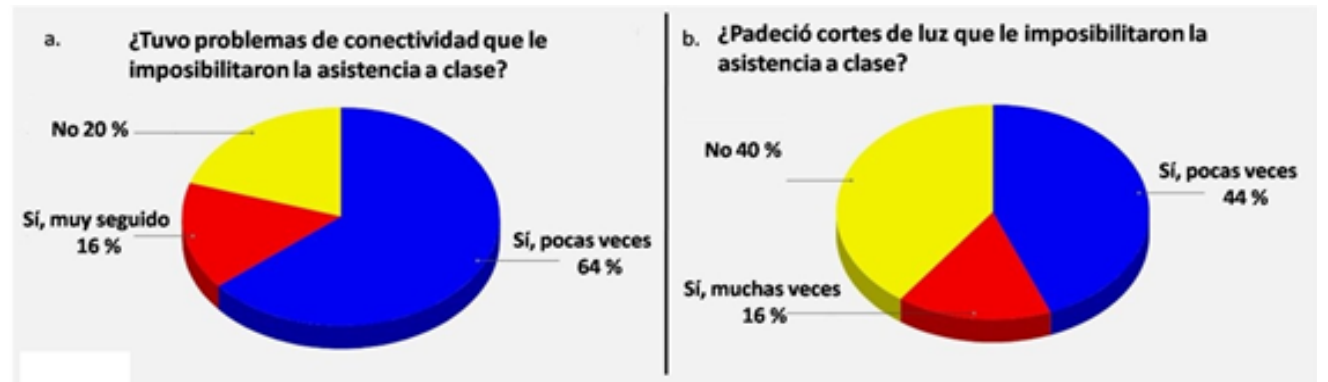

Figura 2. Distribución de la muestra según el porcentaje de alumnos con: a) problemas de conectividad y b) suministro eléctrico durante el dictado de las clases virtuales. 
El 96\% respondió que contaba con los dispositivos tecnológicos necesarios para presenciar las clases virtuales. La mayoría empleó celular y/o notebook (56\%) y en menor proporción una computadora de escritorio para presenciar las clases virtuales y el $52 \%$ compartió sus dispositivos con otra persona. Para el acceso a internet los alumnos emplearon una red hogareña o datos móviles (96\%) y en menor proporción una red pública gratuita (solo el $4 \%$ ). La conectividad fue calificada como buena por el $60 \%$ de los encuestados, como regular por el $16 \%$ y el $24 \%$ tuvo mala conexión. En cuanto a si tuvo problemas de conectividad que le imposibilitaron la asistencia a clase sólo el $16 \%$ tuvo inconvenientes muy seguido, el $64 \%$ pocas veces y el $20 \%$ nunca. En cuanto al suministro eléctrico el $16 \%$ manifestó que tuvo cortes de luz muchas veces, el $44 \%$ pocas veces y el $40 \%$ nunca (Figura 2).

El 88\% no requirió ninguna capacitación ni ayuda para el uso de la tecnología mientras que el $12 \%$ si y realizó capacitación por su cuenta. En cuanto a la información proporcionada en el aula virtual al $68 \%$ le pareció suficiente y sobre la información para realizar los exámenes parciales al $80 \%$ la consideró suficiente y adecuada. El $72 \%$ se encontró satisfecho con el dictado de la materia en las condiciones impuestas por la pandemia Covid-19. Contrariamente el $28 \%$ se mostró insatisfecho. Solo el $24 \%$ de los encuestados consideró beneficiosa esta nueva modalidad virtual de dictado de clases, un gran porcentaje (el 64\%) la calificó como deficiente y un $12 \%$ no tuvo opinión al respecto (Figura 3).

\section{Discusión}

El análisis de los datos recolectados nos proporcionó una información muy valiosa y reveladora. El hecho de que los alumnos vivan con su familia u otras personas afectó su concentración al momento de presenciar las clases virtuales y alrededor de una cuarta parte de los encuestados expresó no contar con un espacio físico apropiado.

El aula se trasladó al hogar del estudiante que no es un ambiente preparado ni cuenta con la infraestructura para el desarrollo de la clase y donde el alumno muchas veces no tiene acceso a los recursos tecnológicos para realizar las actividades a distancia9. Aunque todos los alumnos tenían un dispositivo y conexión a internet solo algo menos de la mitad de los estudiantes calificó positivamente la conectividad. Si bien todos los alumnos son nativos digitales pertenecientes a la generación multimedia, alrededor del diez por ciento de la población estudiantil encuestada no tuvo las competencias digitales para el uso de la tecnología y debió capacitarse por su cuenta. A pesar de que gran parte de los estudiantes ( $3 / 4$ partes) se mostró satisfecho con el desarrollo de la asignatura en las condiciones impuestas por la pandemia Covid-19, más de la mitad lo calificó como deficiente.

Estos datos nos permiten inferir que si bien los esfuerzos de las instituciones y docentes fueron evidentes hay determinadas situaciones que deben ser conocidas y contempladas en el marco de una enseñanza virtual para que haya equidad e igualdad en las condiciones y oportunidades de aprendizaje en un entorno virtual.

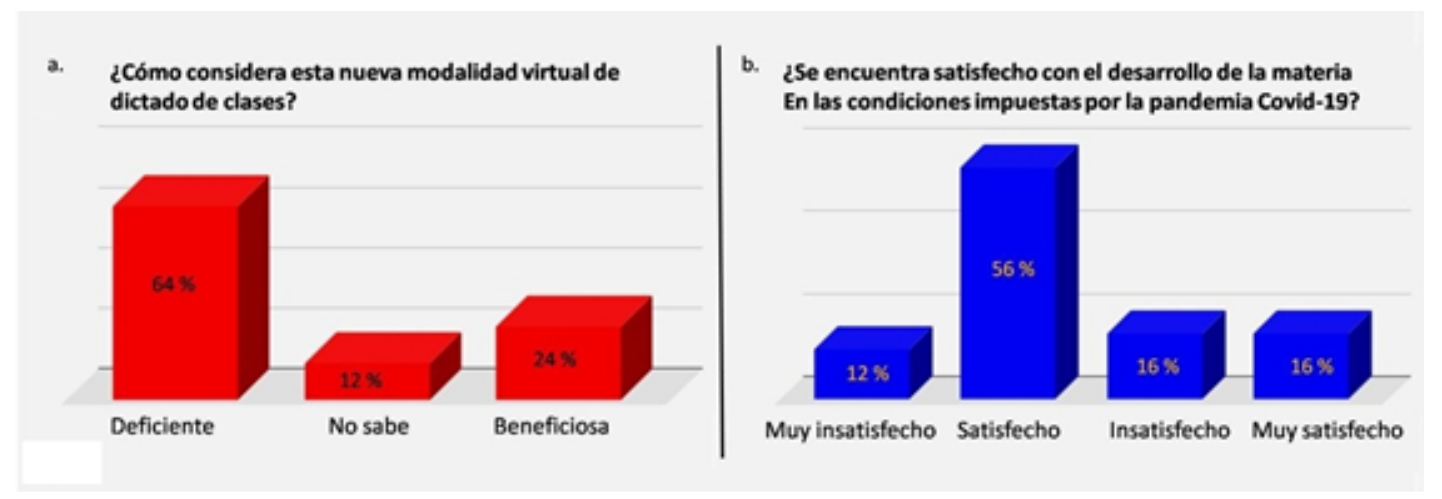

Figura 3. Muestra el porcentaje de alumnos en cuanto a: a) la calificación y b) grado de satisfacción de la modalidad de dictado de clases en tiempos de pandemia Covid-19. 


\section{REFO}

VOL. XIV | N 1| AÑO 2021

ISSN 1668-7280

ISSN-E 2683-7986
Así, nos encontramos ante una situación donde la inclusión de las tecnologías de la información y de la comunicación en la enseñanza universitaria debe entenderse no como una mera adaptación inevitable sino más bien como un proceso enriquecedor, cambiante y en continua evolución donde los alumnos sean protagonistas trabajando interactivamente, construyendo conocimiento y formando parte activa junto a los docentes de un ecosistema comunicativo, integrados a los docentes ${ }^{10,11}$.

La innovación tecnológica, la virtualidad y la educación en línea promueven mayores exigencias para el docente quien debe potenciar al máximo el desarrollo de habilidades digitales y competencias transmedia en sus alumnos ${ }^{2,4,5}$. Las instituciones de educación superior deben establecer un marco que proporcione estrategias integrales y flexibles que permitan la adaptación de los procesos educativos a los grandes cambios socio-tecnológicos de nuestra era ${ }^{12-15}$.

\section{Conclusiones}

La virtualización de la enseñanza universitaria puso en evidencia las dificultades e inconvenientes de la educación en línea debido a la brecha digital producida tanto por la desigual disponibilidad de medios tecnológicos y accesibilidad a internet como así también por las distintas competencias para el uso de las tecnologías de la comunicación y de la información que presentaron estudiantes y docentes.

Es fundamental tomar medidas pertinentes que identifiquen las necesidades y problemas específicos de los estudiantes para eliminar la llamada "brecha digital" y de esta forma estar preparados para los desafíos de la futura educación universitaria post-pandemia, principalmente en lo referente a la inclusión digital y al derecho a la igualdad en las condiciones de aprendizaje de los alumnos donde el estado y sus instituciones juegan un rol trascendental. 


\section{Referencias Bibliográficas}

1. Linne J. Dos generaciones de nativos digitales. Intercom, Rev. Bras. Ciênc. Comun. 2014; 37 (2): 203-221. doi 10.1590/1809-5844 20149

2. Scolari CA. Transmedia literacy in the new media ecology: White paper. H2020 TRANSLITERACY Research Project/Universitat Pompeu Fabra. 2018; 1-16. Disponible en: http://transmedialiteracy.upf.edu/sites/default/files/ files/TL_whit_es.pdf

3. Quevedo, LA. \& Dussel, I. Educación y nuevas tecnologías tecnológicas: los desafíos pedagógicos ante el mundo digital. VI Foro Latinoamericano de Educación. Buenos Aires: Santillana. 2010. Disponible en: https://www.redalyc. org/articulo.oa?id=56741181029

4. III Conferencia Regional de Educación Superior para América Latina y el Caribe Declaración Córdoba, Argentina. 2018; 1-12. Disponible en: https://www.iesalc.unesco. org/wp-content/uploads/2020/08/Declaracion2018PortFinal.pdf

5. LALICS (Latin American Chapter of Globelics). Declaración de Santo Domingo. Foro Regional sobre la Innovación y los Desafíos del Desarrollo de América Latina y el Caribe: retos y oportunidades. República Dominicana. 2017. Disponible en: www.lalics.org

6. Hurtado Olaya PA, García Echeverri M, Rivera Porras DA, Forgiony Santos JO. Las estrategias de aprendizaje y la creatividad: una relación que favorece el procesamiento de la información. Rev ESPACIOS. 2018;39(17):1-18.

7. Camacho JA, Chiappe Laverde A, López de Mesa C. Blended Learning y estilos de aprendizaje en estudiantes universitarios del área de la salud. Rev de Ed Méd Sup. 2012; 26(1):27-44.

8. Wang G, Cheng Z, Yue X, Mc Aleer M. Risk Management of COVID-19 by Universities in China. J. Risk Financial Manag. 2020; 13(2): 36. doi:10.3390/jrfm13020036
9. López Ramírez M., Rodríguez SA. Trayectorias escolares en la educación superior ante la pandemia ¿continuar, interrumpir o desistir? 2020; 66-74. En H. Casanova Cardiel (Coord.), Educación y pandemia: una visión académica Ciudad de México: Universidad Nacional Autónoma de México, Instituto de Investigaciones sobre la Universidad y la Educación. Disponible en: http://132.248.192.241:8080/xmlui/bitstream/handle/ IISUE_UNAM/544/LopezM_RodriguezS_2020_Trayectorias_escolares.pdf?sequence $=1$ \&isAllowed $=y$ 10. Spinelli E. Los modelos de comunicación. Infoamérica. 2009. Disponible en: http://www.infoamerica.org/documentos_pdf/spinelli01.pdf

11. Novomisky S, Manccini G. Tecnologías, medios y procesos pedagógicos: claves para una perspectiva emancipadora. En La Aventura de Innovar con TIC. Aportes para docentes, periodistas y planificadores. La Plata, Argentina: Editorial de Periodismo y Comunicación. 2018.

12. Comunicación e información. Formación en capacitación en información y medios de comunicación. UNESCO. Disponible en: http://www.unesco.org/new/fileadmin/ MULTIMEDIA/HQ/Cl/Cl/pdf/Events/mil_five_laws_spanish.png

13. Ramírez Castañeda LA, Sepúlveda López JJ. Brecha digital e inclusión digital: fenómenos socio - tecnológicos. Revista EIA. 2018; 15 (30): 89-97. https://doi. org/10.24050/reia.v15i30.1152

14. González-Zabala MP, Galvis Lista EA, Sánchez Torres JM. Identificación de factores que afectan el desarrollo de la inclusión digital. Revista Virtual Universidad Católica del Norte. 2015; 44:175-191. Recuperado de http:// revistavirtual.ucn.edu.co/index.php/RevistaUCN/article/ view/623/1158

15. Cabero J, Ruiz-Palmero J. Las Tecnologías de la información y la comunicación para la inclusión: reformulando la brecha digital. IJERI. 2018; 9: 16-30. 